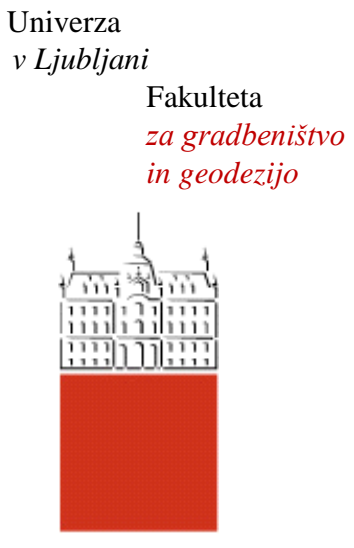

Jamova cesta 2

1000 Ljubljana, Slovenija

http://www3.fgg.uni-lj.si/

\section{DRUGG - Digitalni repozitorij UL FGG http://drugg.fgg.uni-lj.si/}

Ta članek je avtorjeva zadnja recenzirana različica, kot je bila sprejeta po opravljeni recenziji.

Prosimo, da se pri navajanju sklicujte na bibliografske podatke, kot je navedeno:
University
of Ljubljana

Faculty of Civil and Geodetic Engineering

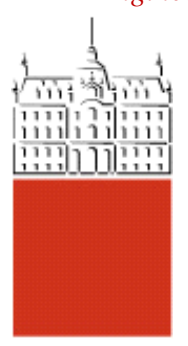

Jamova cesta 2 SI - 1000 Ljubljana, Slovenia http://www3.fgg.uni-lj.si/en/

DRUGG - The Digital Repository http://drugg.fgg.uni-lj.si/

This version of the article is author's manuscript as accepted for publishing after the review process.

When citing, please refer to the publisher's bibliographic information as follows:

Rodman, U., Saje, M., Planinc. I., Zupan, D. 2008. Exact buckling analysis of composite elastic columns including multiple delamination and transverse shear Engineering Structures 30,6: 1500-1514. DOI: 10.1016/j.engstruct.2007.10.003. 


\title{
Exact buckling analysis of composite elastic columns including multiple delamination and transverse shear
}

\author{
U. Rodman, M. Saje, I. Planinc and D. Zupan* \\ University of Ljubljana, Faculty of Civil and Geodetic Engineering, Jamova 2, \\ SI-1115 Ljubljana, Slovenia
}

\begin{abstract}
The exact analytical solution of buckling in beams with multiple delaminations is presented. In order to investigate analytically the influence of axial and shear strains on buckling loads the geometrically exact beam theory is employed with no simplification of the governing equations. The critical forces are then obtained by the linearized stability theory. The parametric studies are designed so that they give us fundamental understanding of the effects of the delamination number, length and position on the buckling load. The effect of shear is found to be of substantial importance.
\end{abstract}

Key words: buckling, multiple delaminations, transverse shear, consistent linearization, composite beam

\section{Introduction}

Composite materials are widely used in industry. The design of composite structures requires good understanding of the behaviour of composite structures and the mechanism of their collapse. One of the failure modes that often takes place in laminated composite structures is the delamination. It is caused by an air entrapment, a local lack of resin or other defects originating from a technological procedure, an impact or a high stress concentration. If compressed the delaminated structure might buckle at a considerably reduced force. That is why the mathematical modelling of various types of delaminations has received a considerable attention during the last decade, see, e.g. the

* Corresponding author. E-mail address: dejan.zupan@fgg.uni-lj.si 
publications by Chen [2] [3], Kardomateas and Schumueser [9], Lim and Parsons [15], Moradi and Taheri [16], Numayr and Haddad [17], MSRao et al. [20], MSRao and Shu [21], Sheinman and Soffer [25], Suemasu [27], Wang et al. [30].

The first attempt in modelling a single-delaminated beam was made by Chai et al. [1], who studied both the stability and the delamination growth by employing the energy release rate criterion. The beam was divided into four regions and the continuity conditions at the delamination ends were applied. A similar model was proposed by Simitses et al. [28] and applied in assessing the effects of the delamination length and position. Later on Yin et al. [31] proposed a simple model for predicting the ultimate load capacity of a singledelaminated beam.

Further work in the delamination theory was focused into multiple delaminations, which is characteristic of laminated composite structures. Most of the early research in multi-delaminated beams and plates relied on numerical methods. Lim and Parsons [15] employed an energy method and assumed displacements to derive the solution. Kutlu and Chang [12] employed the finite element method and also performed experimental studies to predict buckling and post-buckling behaviour of composite plates with two delaminations. Hwang and Liu [8] studied different types of multiple delaminations regarding their effect on buckling behaviour using the finite-element code. Delaminations were assumed centric, equally spaced, but with different lengths. Exact, analytically derived solutions were proposed only recently. Shu [26] performed an exact buckling analysis of a beam with the double delamination. Huang and Kardomateas [7] derived a closed form solution for predicting the buckling load of a composite beam with multiple central delaminations. In both cases, the classical beam theory was used, where the transverse shear effect was neglected. Numayr and Haddad [17] analytically resolved the beam with two delaminations by strictly considering the coupling between the extensional and bending stiffnesses. Normalized axial and bending stiffnesses were proposed by MSRao et al. [20] as a suitable nondimensionalization in case of composite beams made of different materials. An interesting study of multi-delaminated beams was presented by Lee et al. [13] where the buckling analysis is combined with the analysis of the change of the natural frequency of the beam. The longitudinal asymmetry of delamination was recently studied in several papers. MSRao, Song and Shu [22] studied tri-layered Euler-Bernoulli beams with overlapped delaminations. Rao, Wenge and Shu [23] presented the exact solution for Euler-Bernoulli beam with enveloped delaminations. MSRao et al. [20] investigated the beams with asymmetric double separated delaminations.

The effect of the transverse shear effect is only rarely considered in delamination theories, although the inclusion of the transverse shear can reduce the buckling load for a factor proportional to the elastic-to-shear modulus ratio. 
In contrast to standard isotropic materials, where the elastic-to-shear modulus ratio is about two to three, the ratio is often considerably greater for composite materials. Motivated by this consideration, Kardomateas and Schumueser [9] and Chen [2] very early incorporated the transverse shear effect into their studies. They both employed the Griffith-type fracture criterion for studying the onset of the delamination and its subsequent growth. Later on Chen [3] used the first-order shear deformation theory to develop closed-form expressions for buckling and post-buckling of asymmetrically delaminated beams with the clamped boundary. Moradi and Taheri [16] solved the same problem by the differential quadrature method.

In the present paper we derive the exact analytical solution for the buckling load of a multiple asymmetrically delaminated beam. In contrast to the above mentioned authors, our starting point is Reissner's beam theory [24]. We employ the linearized stability theory [10]. The linearized equations are solved in a closed analytical form without any need of simplification of the governing equations. The exact analytical buckling loads are than obtained for a number of boundary conditions. The post-buckling analysis is not the issue of the present paper. The exactness of the proposed approach represents a suitable ground for studying the influence of various parameters. The parametric studies, performed in the present paper, are designed so that they give us fundamental understanding of the effects of the delamination number, length and position on the buckling load. The effect of shear is found to be of substantial importance.

\section{Model}

We consider a straight beam with multiple delaminations under compressive axial forces (Fig. 1). The beam with a constant rectangular cross-section of width $b$ and height $d_{1}$ is laminated with $n$ parallel delaminations which divide the middle part into $n+1$ beam elements. The beam of length $L$ is divided into three parts. $L_{1}$ is the length of the first undelaminated part, $L_{d}(i=$ $2, \ldots, n+2)$ represents the length of the delaminated region and is equal for all delaminations; the length of the remaining region is labeled $L_{n+3}$. If the delaminated region is located symmetrically, $L_{1}=L_{n+3}$. In the asymmetric delaminations, we introduce the ratio, $L_{1 a} / L_{1 s}$, between the length of the first asymmetric element $\left(L_{1}=L_{1 a}\right)$ and the length of the first element in the comparative symmetric case $\left(L_{1 s}=L_{1}\right)$. The thickness of lamina $i$ ( $i=$ $2, \ldots, n+2)$ is defined as $d_{i}$, its relative vertical position with respect to the edge is denoted by $r_{i}$ (Fig. 1). For the sake of clearness, we further introduce the relative length of the delaminations as $l_{d}=L_{d} / L$. 


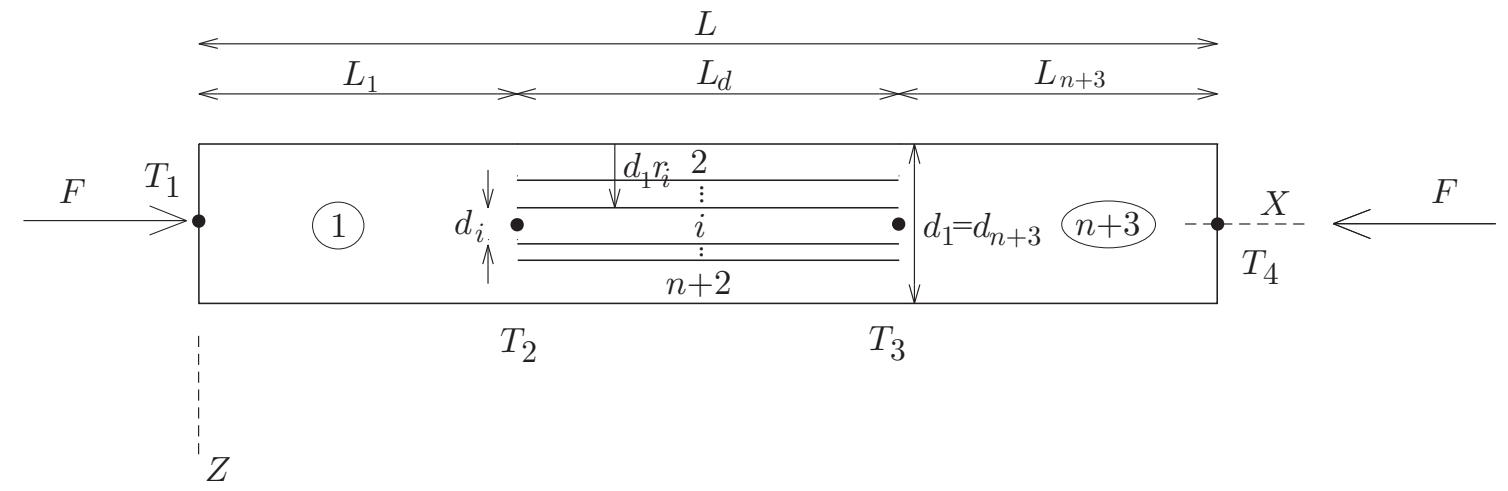

Fig. 1. Model of a multi-delaminated beam with $n$ delaminations.

The slenderness ratio of the beam, $\lambda$, is defined as:

$$
\lambda=L \sqrt{\frac{A}{I_{y}}}
$$

where $A$ denotes the area of the cross-section $\left(A=b d_{1}\right)$ and $I_{y}$ is its centroidal moment of inertia $\left(I_{y}=b \frac{d_{1}^{3}}{12}\right)$. The material of the beam is taken to be linear elastic and described by elastic modulus $E$ and shear modulus $G$. In order to assess the effect of shear, the shear-to-elastic modulus ratio, $G / E$, has been introduced. Note that $G / E$ is strongly dependent on the type of material used:

- $G / E=\infty$; for shear-stiff materials (normally used in the classical theories of delamination),

- $G / E \cong \frac{1}{2}$; for isotropic materials,

- $G / E \cong \frac{1}{6}$; for composite materials.

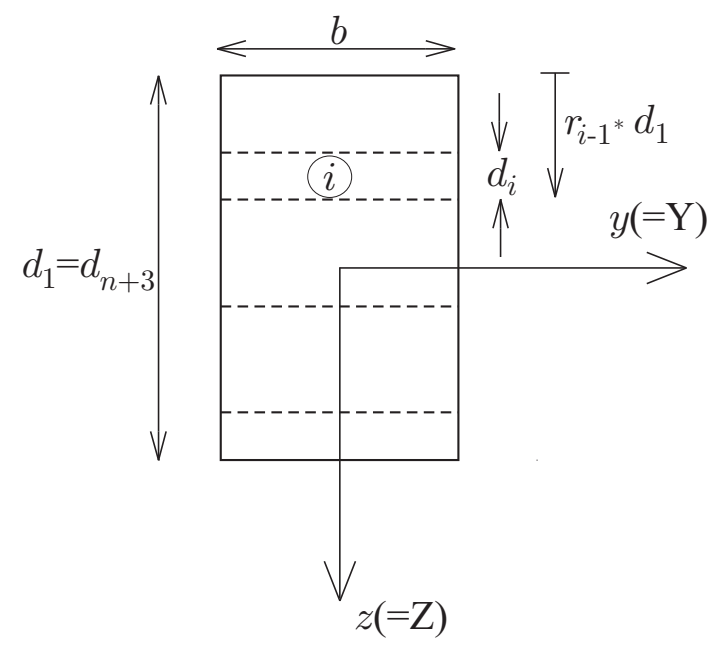

Fig. 2. The cross-section of a delaminated beam with multiple delaminations.

Two Cartesian coordinate systems are introduced. The global coordinate system $(X, Y, Z)$ is chosen, such that the beam lies in the plane $X Z$, with the 
$X$-axis being the centroidal axis of the undeformed beam, and the reference point $(0,0,0)$ coinciding with point $T_{1}$ (Fig. 1 ). The local coordinate system $(x, y, z)$ is assumed to coincide with the global coordinates initially, and then follows the deformation of the beam. The plane cross-sections are assumed to remain planar and to preserve their shape and area during the deformation.

Various boundary conditions will be taken into account: a simply supported beam, a cantilever beam, a clamped-clamped beam, and a clamped-pinned beam. Our goal is to find the smallest axial load such that the buckling occurs.

Note that the present model assumes that the delaminated layers deform freely and have different transverse deformations. This assumption may not be practical due to the possible overlapping of the delaminated layers [29]. In the present paper we will, however, limit the studies to the beams with such types of delaminations that overlapping does not occur or is insignificant. The overlapping of the delaminated layers needs to be avoided in the post-buckling analysis which is not the issue of the present paper. For lower and upper bounds of the buckling load of composite beams with two non-overlapping delaminations see the paper by Parlapalli and Shu [19].

\section{Formulation}

\subsection{Governing equations}

The present stability analysis is based on the exact analytical solution of the linearized planar beam theory [11]. Our starting point is the non-linear Reissner beam theory [24] whose governing equations consist of [4], [5]:

i) kinematic equations

$$
\begin{aligned}
1+u^{\prime} & =(1+\varepsilon) \cos \varphi+\gamma \sin \varphi \\
w^{\prime} & =-(1+\varepsilon) \sin \varphi+\gamma \cos \varphi \\
\varphi^{\prime} & =\kappa,
\end{aligned}
$$

ii) equilibrium equations

$$
\begin{aligned}
R_{X}^{\prime}+p_{X} & =0 \\
R_{Z}^{\prime}+p_{Z} & =0 \\
M^{\prime}-(1+\varepsilon) Q+\gamma N-m_{Y} & =0
\end{aligned}
$$


where

$$
\begin{aligned}
& N=R_{X} \cos \varphi-R_{Z} \sin \varphi \\
& Q=R_{X} \sin \varphi+R_{Z} \cos \varphi
\end{aligned}
$$

iii) and constitutive equations

$$
\begin{aligned}
N & =E \int_{A}(\varepsilon+z \kappa) d A \\
Q & =G A_{s} \gamma \\
M & =E \int_{A} z(\varepsilon+z \kappa) d A .
\end{aligned}
$$

Here $A_{s}$ is the effective shear area, $u$ and $w$ denote the displacements of the axis of the beam, $\varphi$ is the rotation of the cross section, $\varepsilon$ is the extensional strain, $\gamma$ is the shear strain, $\kappa$ is the bending strain (curvature), $p_{X}, p_{Z}$ and $m_{Y}$ are external distributed forces and moments, $R_{X}, R_{Z}$ and $M$ are the stressresultant forces and moment. When expressed with respect to the local basis, the stress-resultant forces are denoted by $N$ and $Q$ and related to $R_{X}$ and $R_{Z}$ via Eqs. (7) and (8).

\subsection{Linearized equations}

As in the paper by Zupan and Saje [32] for three-dimensional beams, a consistent variation of Eqs. (1)-(11) is employed at an arbitrary configuration of the beam. The deduction of the variations needs the variations of the constitutive equations, i.e.

$$
\begin{aligned}
\delta N & =C_{11} \delta \varepsilon+C_{12} \delta \kappa \\
\delta M & =C_{21} \delta \varepsilon+C_{22} \delta \kappa
\end{aligned}
$$

where

$$
\begin{aligned}
& C_{11}=\frac{\partial N}{\partial \varepsilon}=E \int_{A} \frac{\partial}{\partial \varepsilon}(\varepsilon+z \kappa) d A=E A \\
& C_{12}=\frac{\partial N}{\partial \kappa}=E \int_{A} \frac{\partial}{\partial \kappa}(\varepsilon+z \kappa) d A=E \int_{A} z d A=E S_{y} \\
& C_{21}=\frac{\partial M}{\partial \varepsilon}=E \int_{A} \frac{\partial}{\partial \varepsilon}\left(z \varepsilon+z^{2} \kappa\right) d A=E \int_{A} z d A=E S_{y}=C_{12} \\
& C_{22}=\frac{\partial M}{\partial \kappa}=E \int_{A} \frac{\partial}{\partial \kappa}\left(z \varepsilon+z^{2} \kappa\right) d A=E \int_{A} z^{2} d A=E I_{y}
\end{aligned}
$$

are the components of the tangent constitutive matrix of the cross-section. Here $S_{y}$ denotes the moment of area and $I_{y}$ the moment of inertia. Note that $S_{y}$ is not zero, if the centroidal axis does not coincide with the neutral $X$-axis 
of the whole beam. It is suitable to introduce the notation

$$
d=\frac{C_{11} C_{22}-C_{12} C_{21}}{C_{11}}
$$

$C_{11} C_{22}-C_{12} C_{21}$ is the determinant of the constitutive tangent matrix of the cross-section. Both the determinant and the axial stiffness, $C_{11}$, are taken to be strictly positive quantities in our studies.

Assuming that the column to be considered straight and subjected only to axial point force, and varying the non-linear equations of the beam (1)-(11) about an equilibrium state gives

$$
\begin{aligned}
\delta u^{\prime}-\delta \varepsilon & =0 \\
\delta w^{\prime}+(1+\varepsilon) \delta \varphi-\delta \gamma & =0 \\
\delta \varphi^{\prime}-\delta \kappa & =0 \\
\delta R_{X}^{\prime} & =0 \\
\delta R_{Z}^{\prime} & =0 \\
\delta M^{\prime}+R_{X} \delta w^{\prime}-(1+\varepsilon) \delta R_{Z} & =0 \\
C_{11} \delta \varepsilon+C_{12} \delta \kappa-\delta R_{X} & =0 \\
G A_{s} \delta \gamma-R_{X} \delta \varphi-\delta R_{Z} & =0 \\
C_{21} \delta \varepsilon+C_{22} \delta \kappa-\delta M & =0
\end{aligned}
$$

Eqs. (19)-(24) represent a system of six ordinary differential equations for nine unknown functions of $x: \delta u, \delta w, \delta \varphi, \delta R_{X}, \delta R_{Z}, \delta M$. Algebraic equations (25)-(27) represent the linearized constitutive equations yielding the relations between $\delta R_{X}, \delta R_{Z}, \delta M$ and $\delta \varepsilon, \delta \gamma, \delta \kappa$. Owing to the simple form of the total set of equations (19)-(27), it will be easy to find the analytical solution.

\subsection{The analytical solution of linearized equations}

The set of nine equations (19)-(27) can be recast into a system of two higherorder differential equations for axial and lateral deflections $\delta w$ and $\delta u$. After taking the first derivative of Eqs. (20) and (26) with respect to $x$, and considering Eq. (23), we have

$$
\delta w^{\prime \prime}=\left[-(1+\varepsilon)+\frac{R_{X}}{G A_{s}}\right] \delta \varphi^{\prime}=\left[-(1+\varepsilon)+\frac{R_{X}}{G A_{s}}\right] \delta \kappa
$$

and

$$
\delta w^{(i v)}=\left[-(1+\varepsilon)+\frac{R_{X}}{G A_{s}}\right] \delta \kappa^{\prime \prime} .
$$


The second derivative of (27) with respect to $x$ gives

$$
\delta M^{\prime \prime}=C_{21} \delta \varepsilon^{\prime \prime}+C_{22} \delta \kappa^{\prime \prime} .
$$

This is to be compared to the expression, derived from Eqs. (24) and (23):

$$
\delta M^{\prime \prime}=-R_{X} \delta w^{\prime \prime} .
$$

Equating the right hand sides in (30) and (31) gives

$$
C_{21} \delta \varepsilon^{\prime \prime}+C_{22} \delta \kappa^{\prime \prime}+R_{X} \delta w^{\prime \prime}=0 .
$$

Finally, after inserting (25) and (29) into (32) and considering (18), we get

$$
\delta w^{(i v)}+\frac{R_{X}}{d}\left[-(1+\varepsilon)+\frac{R_{X}}{G A_{s}}\right] \delta w^{\prime \prime}=0 .
$$

We assume a compressive axial force; consequently, $R_{X}$, as well as the bracketed term, are negative quantities. It is convenient to introduce the buckling parameter $k^{2}$ as

$$
k^{2}=-\frac{R_{X}}{d}\left[(1+\varepsilon)-\frac{R_{X}}{G A_{s}}\right] .
$$

Hence the fourth order differential equation (33) can be written in a simple form as:

$$
\delta w^{(i v)}+k^{2} \delta w^{\prime \prime}=0 .
$$

Eq. (35) can be solved analytically, the solution being

$$
\delta w(x)=A \sin k x+B \cos k x+C x+D .
$$

Taking the first derivative of (25) with respect to $x$ and considering (19) and (22) yields

$$
C_{11} \delta u^{\prime \prime}+C_{12} \delta \kappa^{\prime}=0 .
$$

From (28) we then obtain

$$
\frac{C_{11}}{C_{12}}\left[-(1+\varepsilon)+\frac{R_{X}}{G A_{s}}\right] \delta u^{\prime \prime}+\delta w^{\prime \prime \prime}=0 .
$$

After inserting $\delta w$ from Eq. (36) and taking into account (34), we are left with the second-order differential equation for $\delta u$ :

$$
\delta u^{\prime \prime}=\frac{R_{X} C_{12}}{d C_{11}} k(A \cos k x-B \sin k x),
$$

whose solution reads

$$
\delta u(x)=\alpha+\beta x-\frac{R_{X} C_{12}}{k d C_{11}}(A \cos k x-B \sin k x) .
$$


An arbitrary deformed configuration of the linearized beam is uniquely described by $\delta u(x), \delta w(x)$.

It is obvious from Eqs. (36) and (38) that the analytical solution for the displacements of a planar beam element under axial point forces has 6 parameters, $A, B, C, D, \alpha$, and $\beta$. They are determined from the boundary and continuity conditions of each element. Due to the general approach employed above it is obvious that the form of solutions (36) and (38) holds for any of $n+3$ elements. Each element has, however, different parameters $A_{i}, B_{i}, C_{i}$, $D_{i}, \alpha_{i}$ and $\beta_{i}, i=1, \ldots, n+3$. After the parameters have been obtained, the remaining functions $\delta \varphi, \delta R_{X}, \delta R_{Z}, \delta M$ of each element can be obtained by inserting the solutions (36) and (38) into Eqs. (19)-(27).

From (24) we have

$$
\delta R_{Z}=\frac{R_{X}}{1+\varepsilon} \delta w^{\prime}+\frac{1}{1+\varepsilon} \delta M^{\prime} .
$$

$\delta M^{\prime}$ can be expressed by Eqs. (27) and (25) as

$$
\delta M^{\prime}=d \delta \kappa^{\prime}
$$

Using Eqs. (40) and (29) in (39) yields

$$
\delta R_{Z}=\frac{R_{X}}{1+\varepsilon}\left(\delta w^{\prime}+\frac{1}{k^{2}} \delta w^{\prime \prime \prime}\right) .
$$

Inserting the solution (36) for $\delta w$ into (41) results in

$$
\delta R_{Z}=\frac{R_{X}}{1+\varepsilon} C
$$

From Eq. (19) we have

$$
\delta \gamma=\delta w^{\prime}+(1+\varepsilon) \delta \varphi
$$

employing Eq. (26) and rearranging terms we finally get

$$
\delta \gamma=\frac{R_{X}}{G A_{s}} \delta \varphi+\frac{1}{G A_{s}} \delta R_{Z}
$$

Upon inserting Eqs. (44) and (41) into (43) we derive

$$
\delta \varphi=-\frac{1}{1+\varepsilon}\left[\delta w^{\prime}+\frac{R_{X}^{2}}{G A_{s} k^{4} d} \delta w^{\prime \prime \prime}\right] .
$$

Finally, after we insert the solution (36) into (45) and rearrange the terms, we 
obtain

$$
\delta \varphi=\frac{R_{X}}{k d}(A \cos k x-B \sin k x)-\frac{1}{1+\varepsilon} C .
$$

By inserting (19) and (28) into (27) we obtain

$$
\delta M=C_{21} \delta u^{\prime}+C_{22} \frac{R_{X}}{k^{2} d} \delta w^{\prime \prime}
$$

and, in an analogous way,

$$
\delta R_{X}=C_{11} \delta u^{\prime}+C_{12} \frac{R_{X}}{k^{2} d} \delta w^{\prime \prime} .
$$

By inserting solutions (36)-(38) into (47) and (48) and after a straightforward derivation we finally have

$$
\begin{aligned}
& \delta R_{X}=C_{11} \beta \\
& \delta M=C_{21} \beta-R_{X}(A \sin k x+B \cos k x) .
\end{aligned}
$$

\section{Boundary and continuity conditions}

The boundary conditions of $n+3$ elements constituting the multi-delaminated beam are divided into:

i) boundary conditions at both ends of the beam, as enforced by the supports, and

ii) the continuity conditions of displacements and stresses at the contacts of ideal and delaminated parts of the beam.

Before we discuss the conditions to be imposed on the linearized formulation, the continuity of displacements and the equilibrium of forces in non-linear primary configuration need to be considered. The requirements of continuity of the displacements at the ends of the delaminated portion of the beam read

$$
\begin{gathered}
u_{1}\left(L_{1}\right)=u_{i}(0), \quad \text { for } i=2, \ldots, n+2 \\
u_{i}\left(L_{d}\right)=u_{n+3}(0), \quad \text { for } i=2, \ldots, n+2 .
\end{gathered}
$$


For an initially straight beam subjected to an axial load, Eq. (1) gives

$$
\begin{gathered}
u^{\prime}=\varepsilon \\
u(x)=u(0)+\varepsilon x .
\end{gathered}
$$

Upon inserting (53) into (52) we have the condition

$$
u_{1}\left(L_{1}\right)+\varepsilon_{i} L_{d}=u_{n+3}(0),
$$

which requires the equality of axial strains of the layers:

$$
\varepsilon_{i}=\varepsilon_{j}, \quad i, j \in 2, \ldots, n+2 .
$$

Hence the constant strain of the layers is denoted by $\varepsilon_{d}$. Equilibrium conditions of axial forces at the contact of the delaminated and the ideal parts (points $T_{2}$ and $T_{3}$ ) read

$$
\begin{aligned}
\sum_{i=2}^{n+2} R_{X, i}(0) & =R_{X, 1}\left(L_{1}\right) \\
\sum_{i=2}^{n+2} R_{X, i}\left(L_{d}\right) & =R_{X, n+3}(0) .
\end{aligned}
$$

For a straight axially loaded beam, the axial forces are expressed with axial strains as:

$$
R_{X, i}=E A_{i} \varepsilon_{i}, \quad \text { for } i=1, \ldots, n+3 .
$$

By inserting (57) into (55)-(56) and considering (54) we get

$$
\begin{aligned}
& \sum_{i=2}^{n+2} E A_{i} \varepsilon_{d}=E A_{1} \varepsilon_{1} \\
& \sum_{i=2}^{n+2} E A_{i} \varepsilon_{d}=E A_{n+3} \varepsilon_{n+3} .
\end{aligned}
$$

We have assumed the constant cross-section of the column; thus, $\sum_{i=2}^{n+2} A_{i}=$ $A_{1}=A_{n+3}=A$, and

$$
\varepsilon=\varepsilon_{i}=\text { const. }, \quad \text { for } i=1, \ldots, n+3 .
$$

From (57) it follows

$$
R_{X, i}=E A_{i} \varepsilon, \quad \text { for } i=1, \ldots, n+3 .
$$

The equilibrium at the right end gives

$$
R_{X, n+3}=-F
$$

and

$$
\varepsilon=-\frac{F}{E A}
$$


The axial forces in the layers then follow as

$$
\begin{aligned}
& R_{X, i}=-\frac{A_{i}}{A} F, \quad \text { for } i=2, \ldots, n+2 \\
& R_{X, 1}=-F
\end{aligned}
$$

As reported by $\mathrm{Li}$ [14] the exact solution for buckling, if the effect of shear is considered, can not be easily obtained for a non-uniform bar, such as a multi-step bar. In contrast, the present approach allows us to directly extend the formulation to step columns.

At the ends of the delamination region $\left(T_{2}\right.$ and $\left.T_{3}\right)$, the linearized solution should satisfy the continuity conditions for displacements and rotations and the equilibrium of the internal forces, i.e.:

$$
\begin{gathered}
\delta u_{1}\left(L_{1}\right)=\delta u_{i}(0), \quad \text { for } i=2, \ldots, n+2 \\
\delta w_{1}\left(L_{1}\right)=\delta w_{i}(0), \quad \text { for } i=2, \ldots, n+2 \\
\delta \varphi_{1}\left(L_{1}\right)=\delta \varphi_{i}(0), \quad \text { for } i=2, \ldots, n+2 \\
\delta R_{X, 1}\left(L_{1}\right)=\sum_{i=2}^{n+2} \delta R_{X, i}(0) \\
\delta R_{Z, 1}\left(L_{1}\right)=\sum_{i=2}^{n+2} \delta R_{Z, i}(0) \\
\delta M_{1}\left(L_{1}\right)=\sum_{i=2}^{n+2} \delta M_{i}(0) \\
\sum_{i=2}^{n+2} \delta R_{X, i}\left(L_{d}\right)=\delta R_{X, n+3}(0) \\
\sum_{i=2}^{n+2} \delta R_{Z, i}\left(L_{d}\right)=\delta R_{Z, n+3}(0) \\
\sum_{i=2}^{n+2} \delta M_{i}\left(L_{d}\right)=\delta M_{n+3}(0)
\end{gathered}
$$

and

$$
\begin{aligned}
& \delta u_{i}\left(L_{d}\right)=\delta u_{n+3}(0), \quad \text { for } i=2, \ldots, n+2 \\
& \delta w_{i}\left(L_{d}\right)=\delta w_{n+3}(0), \quad \text { for } i=2, \ldots, n+2 \\
& \delta \varphi_{i}\left(L_{d}\right)=\delta \varphi_{n+3}(0), \quad \text { for } i=2, \ldots, n+2 \text {. }
\end{aligned}
$$

In what follows, we analyze only the beams whose one end (point $T_{1}$ ) is fixed in the axial direction, while the other end (point $T_{4}$ ) is subjected to a compressive 
force; hence its variation is zero:

$$
\begin{aligned}
\delta u_{1}(0) & =0 \\
\delta R_{X, n+3}\left(L_{n+3}\right) & =0 .
\end{aligned}
$$

Four different combinations of the boundary conditions will be analyzed (Fig. $3)$ :

(a) Clamped at one end, free at the other (the cantilever beam)

$$
\delta w_{1}(0)=0, \quad \delta \varphi_{1}(0)=0, \quad \delta R_{Z, n+3}\left(L_{n+3}\right)=0, \quad \delta M_{n+3}\left(L_{n+3}\right)=0 .
$$

(b) Pinned at both ends (the simply supported beam)

$$
\delta w_{1}(0)=0, \quad \delta w_{n+3}\left(L_{n+3}\right)=0, \quad \delta M_{1}(0)=0, \quad \delta M_{n+3}\left(L_{n+3}\right)=0 .
$$

(c) Clamped column at one end, pinned at the other

$$
\delta w_{1}(0)=0, \quad \delta \varphi_{1}(0)=0, \quad \delta w_{n+3}\left(L_{n+3}\right)=0, \quad \delta M_{n+3}\left(L_{n+3}\right)=0 .
$$

(d) Clamped at both ends

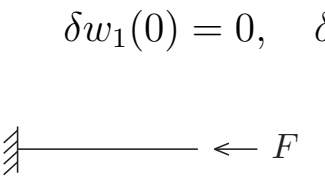

(a) (b)

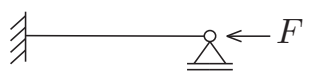

(c)

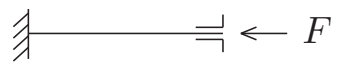

(d)

Fig. 3. Beam models for (a) cantilever, (b) simply supported, (c) clamped-pinned and (d) clamped-clamped beam.

The complete set of equations for $n+3$ elements consists of $6(n+2)$ continuity conditions (64)-(75) and 6 boundary conditions, i.e. totally $6(n+3)$ equations for $6(n+3)$ unknowns: $A_{i}, B_{i}, C_{i}, D_{i}, \alpha_{i}, \beta_{i}, i=1, \ldots, n+3$. We are interested only in non-trivial solutions, where all the parameters are not equal to zero. The equations are linear algebraic and homogenous, so that they can be written in the form

$$
\boldsymbol{K} \boldsymbol{\alpha}=\mathbf{0}, \quad \boldsymbol{\alpha} \neq \mathbf{0}
$$

where $\boldsymbol{K}$ denotes the $6(n+3) \times 6(n+3)$ matrix of coefficients and $\boldsymbol{\alpha}$ the vector of $6(n+3)$ unknowns. We look for the lowest value of the axial load $F$ denoted by $F_{c r}$, which renders the non-trivial solution for $\boldsymbol{\alpha}$. This is achieved only if $\operatorname{det} \boldsymbol{K}=0$. The analytical expressions for $\operatorname{det} \boldsymbol{K}$ are too complicated to be here presented in a closed form. The results and several extensive parametric studies are presented in the next section. 


\section{$5 \quad$ Numerical results}

In this section we compare the results of the present analytical formulation to the results available in literature. Yet we limit our verification study on the beams with single or double delaminations only.

Note that the present theory agrees with the classical approach only if some simplifications are made. To be able to compare adequately the present analytical results with the classical ones, shear-stiff and axially incompressible beam material must be used $(G=\infty, \varepsilon=0)$. The buckling parameter is then defined as $k^{2}=-\frac{R_{X}}{d}$. This case is in the tables which follow referred to as 'sim.' case.

It is convenient to define the normalized buckling load $F_{c r} / F_{\text {eu }}$, where $F_{\text {eu }}$ is Euler's [6] buckling load for the undelaminated beam $\left(F_{e u}=\frac{E I \pi^{2}}{L_{b}^{2}}\right)$. Here $L_{b}$ is Euler's buckling length, which is strongly dependent on the actual boundary conditions.

In Table 1 we show the results for the normalized buckling load for the simplysupported beam with slenderness ratio $\lambda=45$. Elastic modulus $E=3 \cdot 10^{7}$ $\mathrm{N} / \mathrm{m}^{2}$ was assumed. In this case the beam was delaminated with a single longitudinally symmetric delamination. The results are presented for the relative vertical position $r_{d}=0.4$ and various lengths of the delamination $\left(l_{d}=\right.$ $0.2,0.4,0.6,0.8)$, and compared to those, obtained by the Abaqus finite element code [15], Simitses et al. [28] and the energy method proposed by Lim and Parsons [15].

Table 1

Normalized buckling loads for simply supported beam with a single delamination at relative vertical position $r_{d}=0.4$ and various lengths, $l_{d}$.

\begin{tabular}{c|ccc|ccc}
\hline$l_{d}$ & En. method $[15]$ & Abaqus $[15]$ & Simitses et al. $[28]$ & present $(\operatorname{sim})$. & present $(G=\infty)$ & present $(G=E / 6)$ \\
\hline \hline 0.2 & 0.9997 & 0.9997 & 0.9997 & 0.9997 & 1.0081 & 0.9534 \\
0.4 & 0.9902 & 0.9902 & 0.9902 & 0.9902 & 0.9984 & 0.9447 \\
0.6 & 0.9198 & 0.9197 & 0.9198 & 0.9198 & 0.9269 & 0.8803 \\
0.8 & 0.7264 & 0.7264 & 0.7264 & 0.7264 & 0.7308 & 0.7013 \\
\hline
\end{tabular}

If the influence of shear is ignored and the axial deformation is not included in the buckling parameter $\left(G=\infty, k^{2}=-\frac{R_{X}}{d}\right)$, the present results fully agree with others (Table 1 ). If we also include the effect of axial deformations $\left(k^{2}=-(1+\varepsilon) \frac{R_{X}}{d}\right)$, the present results indicate that Euler's buckling force [6] is conservative $\left(F_{c r} / F_{\text {eu }}>1\right)$. The last column of Table 1 displays the buckling loads for an elastic material with a shear modulus, characteristic for a fibreglass material. The results indicate a decrease of the buckling load if compared 
to the shear-stiff case. Thus, the consideration of the transverse shear might be important in such materials.

The next comparison is made for the beam, clamped at both sides, with a single longitudinally symmetric delamination. For the calculation of the normalized buckling loads, we used the same material and geometric characteristics as in the previous case. In Table 2 we compare the present results with the results of Chen [2] ('CLT' - classical lamination theory and 'SDT' - shear deformation theory), MSRao and Shu [21], and Huang and Kardomateas [7]. Again, a very good agreement between the present theory and the classical, 'shear-stiff' results [2] (CLT), [7], [21] can be observed. When the shear effect is considered, the so called SDT by Chen [2] and the present results also agree well. The differences probably stem from the different approaches in considering the shear correction factor.

Table 2

Normalized buckling loads of clamped-clamped beam with a single delamination at two different relative vertical positions $r_{d}=0.2,0.3$ and various lengths $l_{d}$.

\begin{tabular}{c|ccccc|cc}
\hline$l_{d}$ & $r_{2}$ & SDT $[2]$ & CLT [2] & MSRao $[21]$ & Hu. and Kar. [7] & present (sim.) & present $(G=E / 6)$ \\
\hline \hline 0.2 & 0.2 & 0.7816 & 0.9264 & 0.9370 & 0.9264 & 0.9261 & 0.7969 \\
& 0.3 & 0.8280 & 0.9924 & 0.9995 & 0.9924 & 0.9920 & 0.8463 \\
\hline 0.4 & 0.2 & 0.2354 & 0.2471 & 0.2476 & 0.2470 & 0.2470 & 0.2357 \\
& 0.3 & 0.4803 & 0.5314 & 0.5316 & 0.5314 & 0.5313 & 0.4837 \\
\hline 0.6 & 0.2 & 0.1080 & 0.1103 & 0.1105 & 0.1103 & 0.1103 & 0.1080 \\
& 0.3 & 0.2322 & 0.2435 & 0.2438 & 0.2435 & 0.2435 & 0.2325 \\
\hline 0.8 & 0.2 & 0.0615 & 0.0623 & 0.0623 & 0.0623 & 0.0623 & 0.0615 \\
& 0.3 & 0.1353 & 0.1390 & 0.1398 & 0.1390 & 0.1390 & 0.1353 \\
\hline
\end{tabular}

In our last example we consider the case with two delaminations, as presented by Lim and Parsons [15]. The clamped-clamped beam has two delaminations at relative positions $r_{2}=0.3, r_{3}=0.6$. The normalized lengths of the delamination are taken to be $0.2,0.4,0.6,0.8$ and are positioned symmetrically along the length of the beam. Two different shear moduli were considered $(G=\infty$, $G=E / 6)$ and both simplified and exact buckling parameters were used in order to compare the present results with the classical theories. Other material and geometric characteristics are taken the same as in the case with a single delamination.

Results in Table 3 confirm good agreement of the presented method for the given range of the delamination lengths. Only a slight discrepancy is observed for short delaminations $\left(l_{d}=0.2\right)$ which is due to the slenderness effect. The results of the present formulation for $G=E / 6$ are also presented. As previously, the normalized buckling load of a shear-stiff material $(G=\infty)$ is overestimated.

The above results show that the normalized buckling force of the delaminated 
Table 3

Normalized buckling loads of clamped-clamped beam with two delaminations of various lengths at relative vertical positions $r_{2}=0.3$ and $r_{3}=0.6$.

\begin{tabular}{c|cccc|ccc}
\hline$l_{d}$ & En. method [15] & Abaqus [15] & Shu $[26]$ & Hu. and Kar. $[7]$ & present $(\operatorname{sim})$. & present $(G=\infty)$ & present $(G=E / 6)$ \\
\hline \hline 0.2 & 0.8939 & 0.8940 & 0.9835 & - & 0.8914 & 0.9101 & 0.8009 \\
0.4 & 0.5054 & 0.5056 & 0.5057 & 0.5057 & 0.5036 & 0.5105 & 0.4669 \\
0.6 & 0.2374 & 0.2375 & 0.2374 & 0.2374 & 0.2374 & 0.2385 & 0.2310 \\
0.8 & 0.1374 & 0.1375 & 0.1374 & 0.1374 & 0.1374 & 0.1378 & 0.1352 \\
\hline
\end{tabular}

column depends on a number of parameters:

(i) the relative delamination length $l_{d}$;

(ii) the relative vertical position $r_{i}(i=2, \ldots, n+2)$ of the delamination;

(ii) elastic-to-shear modulus ratio $E / G$;

(iv) the slenderness ratio $\lambda$;

(v) the number of delaminations $n$;

(vi) the relative longitudinal position of the delamination;

(vii) boundary conditions.

In what follows we systematically assess the influence of the above parameters on the buckling load.

\section{$6 \quad$ Parametric studies}

\subsection{Effect of the delamination length and its vertical position}

We study the effect of the delamination length and the relative vertical delamination position on the normalized buckling load. The simply supported and clamped-clamped beams with slenderness ratio $\lambda=45$ and elastic modulus $E=3 \cdot 10^{7} \mathrm{~N} / \mathrm{m}^{2}$ have been analyzed. A single longitudinally symmetric delaminations of various length $l_{d}$ are positioned at several vertical positions $r_{2}=0.02,0.05,0.1,0.2,0.3,0.4,0.5$. Note that the relative vertical position also uniquely defines the ratio between the lamina thickness and the beam height: $r_{2}=\frac{d_{2}}{h}$.

Results for the shear-stiff and a realistic composite material are presented in Fig. 4. By the increase of the length of delamination $l_{d}$, the normalized buckling load $F_{c r} / F_{e u}$ is reduced. If the relative vertical position of the delamination is close to the centroid of the cross-section $\left(0.3<r_{2}<0.5\right)$, the decrease of the buckling force is not significant for short delaminations. In contrast, a significant reduction is observed for longer delaminations $\left(l_{d}>0.5\right)$. For the delaminations with a small vertical relative position, $r_{2}<0.3$ (thin 


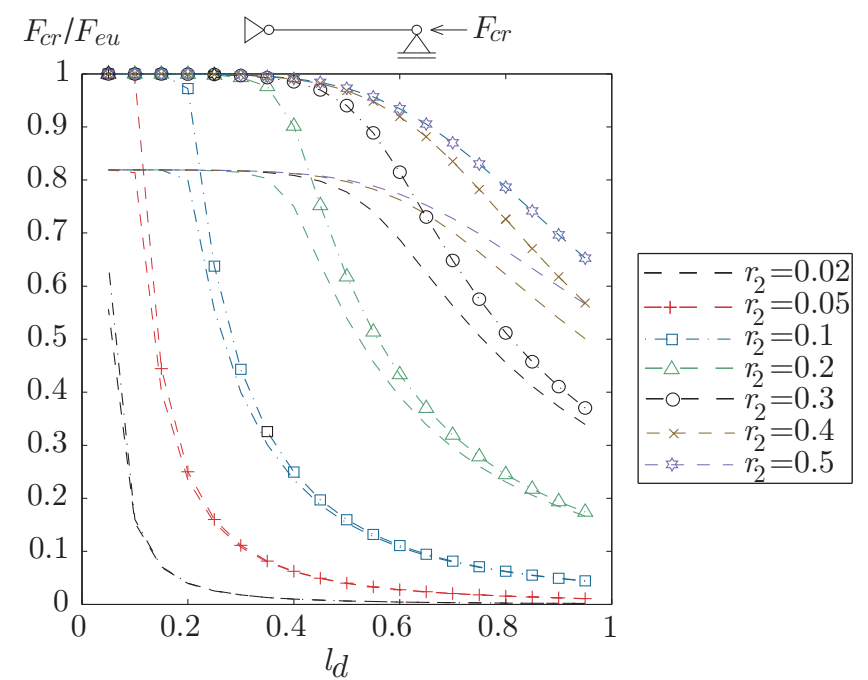

(a)

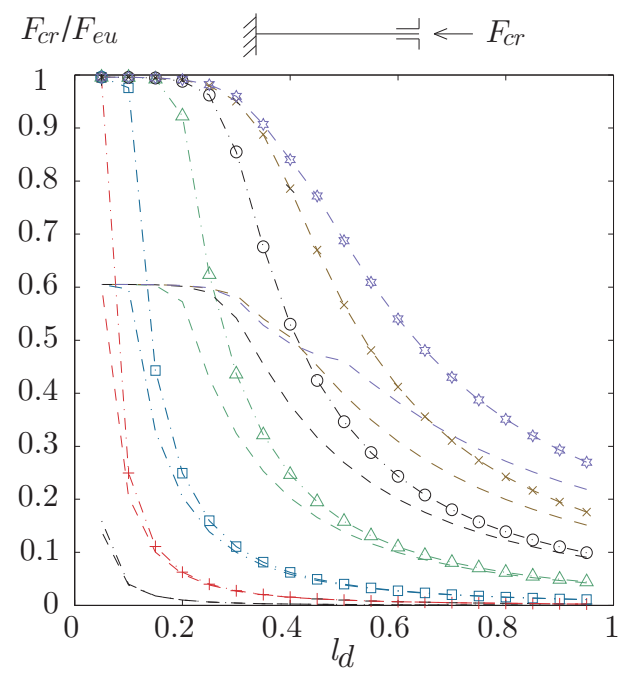

(b)

Fig. 4. Normalized buckling loads for (a) simply supported and (b) clamped-clamped beam with a single delaminaton vs. delamination length $l_{d}$ for various relative positions of delamination $r_{d}$ and two shear moduli $G=E / 6$ (dashed) and $G=\infty$ (marked).

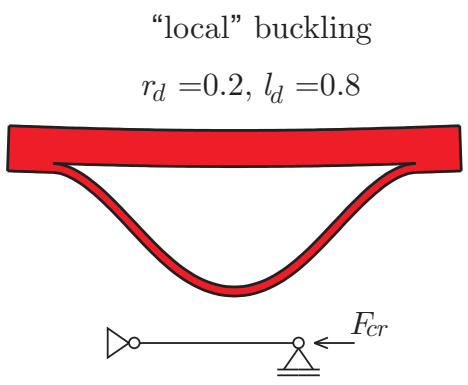

(a)

$$
\text { "mixed" buckling }
$$$$
r_{d}=0.2, l_{d}=0.4
$$

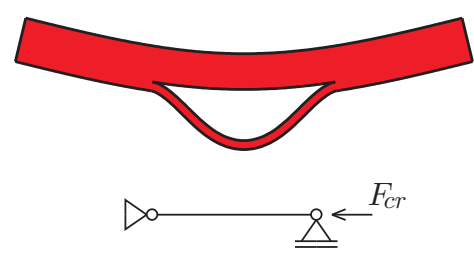

(b) "global" buckling

$r_{d}=0.2, l_{d}=0.2$

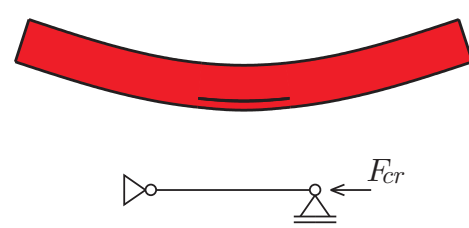

(c)

Fig. 5. Buckling shapes for simply supported beam with single delaminaton for various delamination lengths, $l_{d}$.

delaminations), the reduction of the relative buckling load is substantial even for short delaminations. In order to show the influence of the shear modulus on the buckling load, the results are presented for both shear-stiff material and realistic composite material in the same figure. The shapes of the buckling load vs. the delamination length curves for $G=\infty$ (marked curve) and $G / E=1 / 6$ (dashed curve) are similar, yet a considerable differences in quantitative terms are noticeable. In particular, the normalized ultimate buckling force is significally reduced, when the transverse shear effect is considered. The difference between the buckling loads is even more pronounced for short delaminations 
and higher relative positions of the delamination (thicker laminae). By reducing the shear-to-elastic modulus ratio, this effect is even enhanced. The similar reduction of the relative buckling force due to the transverse shear effect is also noticed for multiple delaminations $(n>1)$.

Both the delamination length and the vertical position affect the buckling shapes of the beam, see Fig. 5. For long and thin delaminations (a), the local buckling of laminae is dominant, unlike for short and thick delaminations (c), where the effect of delamination onto the buckling load is negligible. In some cases both the delamination and the global buckling participate comparably to the buckling of the beam, behaving in the so-called 'mixed buckling'.

\subsection{Effect of shear modulus}

As we have already noticed, the normalized buckling load strongly depends on the value of shear modulus $G$. To evaluate the influence of transverse shear in a more detail, we investigated various delaminated beams with a number of shear moduli. In Figs. 6 and 7 we present normalized buckling loads vs. shearto-elastic modulus ratio for several lengths $\left(l_{d}\right)$ and numbers of delaminations $(n)$. In this case, the longitudinally symmetrical delaminations are equally distributed over the height of the element, so that the thickness $d_{i}$ is equal for all laminae. The number of delaminations therefore fully defines the relative vertical position, $r_{i}$, of each delamination. The beam with slenderness ratio $\lambda=18$ is analyzed for four types of boundary conditions.

From the results in Figs. 6 and 7 we can observe that the transverse shear effect might be of some importance. The influence of shear decreases for beams with longer delaminations and for beams with larger number of delaminations. For a larger number of long delaminations $\left(n=5, l_{d}>0.2\right)$, the shear effect can be neglected. For a single delamination $(n=1)$, the difference between the normalized buckling force between $G=0.1 E$ (fiber-reinforced composite) and $G=0.6 E$ (standard nearly isotropic material such as concrete and steel) is up to $20 \%$ for the simply supported beam and up to $33 \%$ for the clamped beam. The influence of shear decreases with increasing length of the delamination, but it still remains worth of considering, even for longer delaminations. For $l_{d}=0.8$ the difference between the buckling force for $G=0.1 E$ and $G=0.6 E$ is about $10 \%$ for the simply supported beam and up to $8 \%$ for the clampedclamped beam. Generally the most significant influence of shear is observed if beams are clamped on both ends having a single delamination (up to 33\%). From the results given above we can conclude, that the shear might sometimes substantially affect the critical forces of the delaminated beam. 


\subsection{Effect of the slenderness ratio}

The goal is to determine the range of the slendernesses, where the influence of shear modulus is considerable. We present the results for a beam with one and three delaminations. Fig. 8 shows the results for the normalized buckling loads for three different shear-to-elastic modulus ratios $(G / E=0.1,0.33,0.57)$. Two different length of delaminations $\left(l_{d}=0.2,0.5\right)$ were considered in order to observe the effect of slenderness for short and middle-length delaminations.

The results confirm the importance of the transverse shear effect. Namely, the normalized buckling force may decrease by about $20 \%$ for simply supported stocky beams $(\lambda=20)$. Comparison is made between the lowest, $G / E=$ 0.1 , and the largest, $G / E=0.57$ shear-to-elastic modulus ratio. This effect becomes even larger for the clamped-pinned and the clamped-clamped beams.
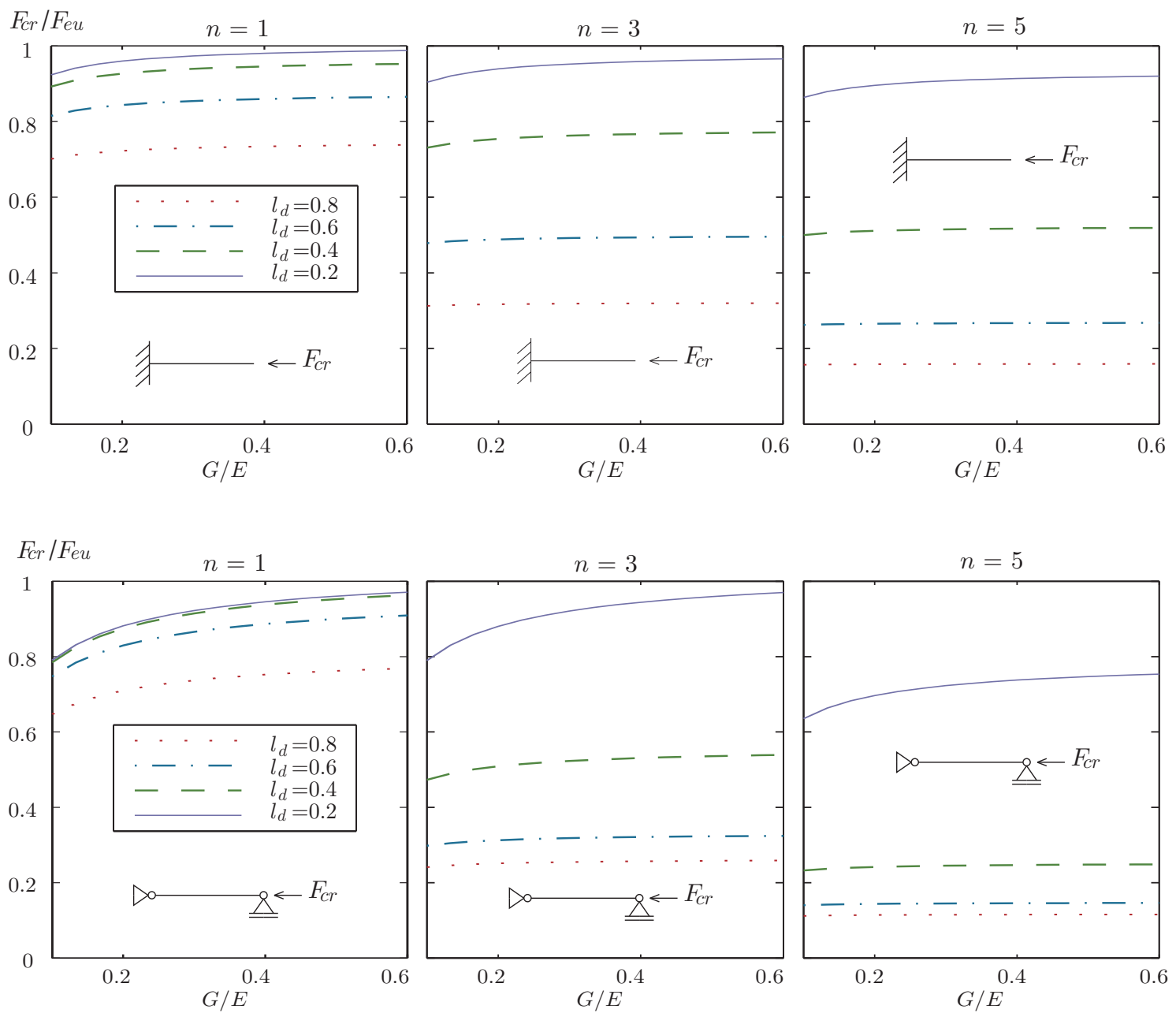

Fig. 6. Effect of transverse shear on the normalized buckling load for the cantilever and the simply supported beam with 1, 3 and 5 delaminations of various lengths. 

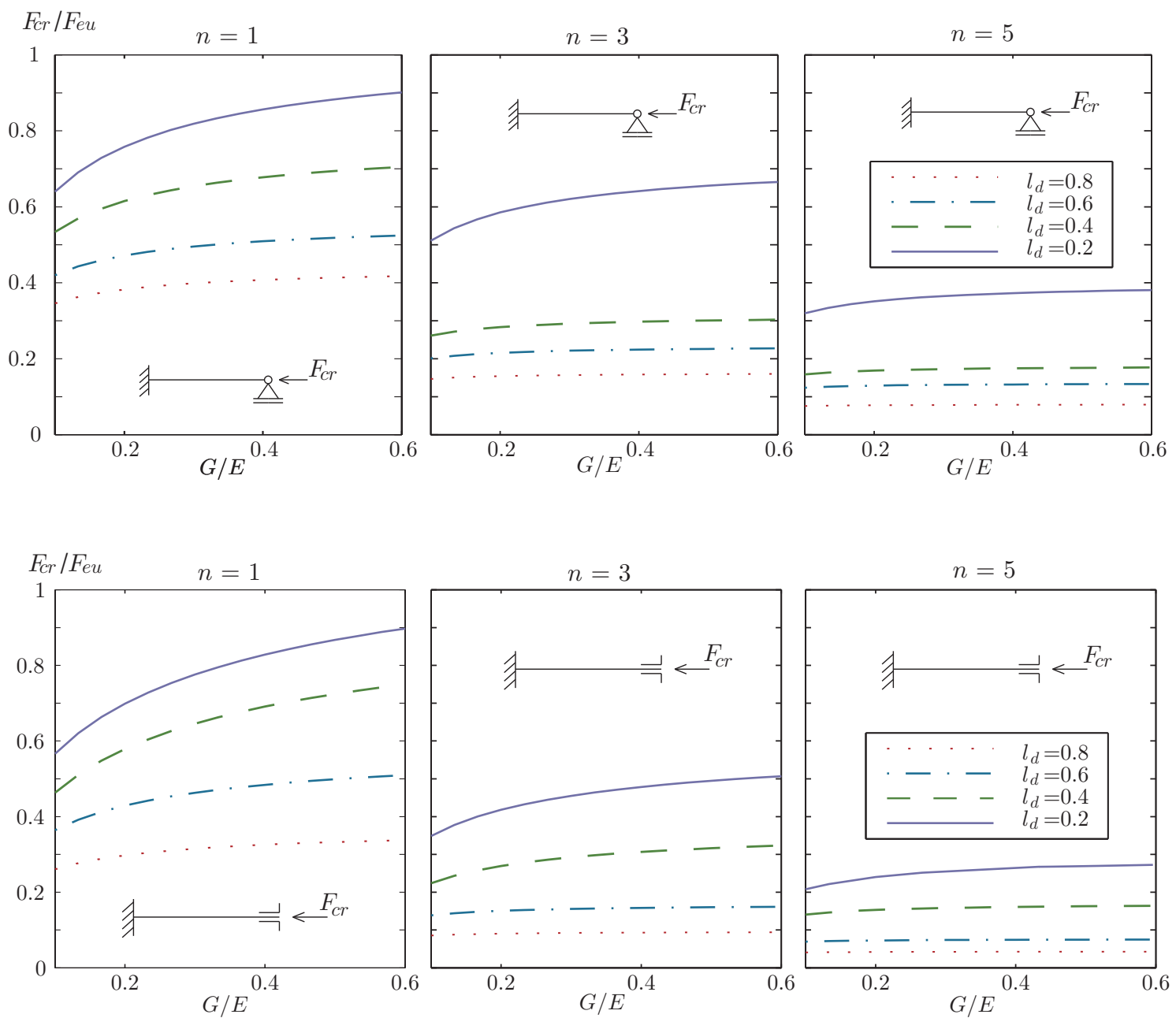

Fig. 7. Effect of transverse shear on the normalized buckling load for the clamped-pinned and the clamped-clamped beams with 1, 3 and 5 delaminations of various lengths, $l_{d}$.

If the beams get more and more slender, the effect of shear decreases; for the clamped-clamped beam, however, it is not negligible even for $\lambda=50$. In principle the same effect, yet only in a smaller scale, can be observed for longer delaminations.

For beams with three delaminations, the shear effect is more influential for stocky beams, but effect of the length of the delamination can this time be enormous. In case of a single delamination $(n=1)$, no such extreme differences could be observed. This gives us the motivation to investigate further the influence of the number of delaminations on the buckling load. 

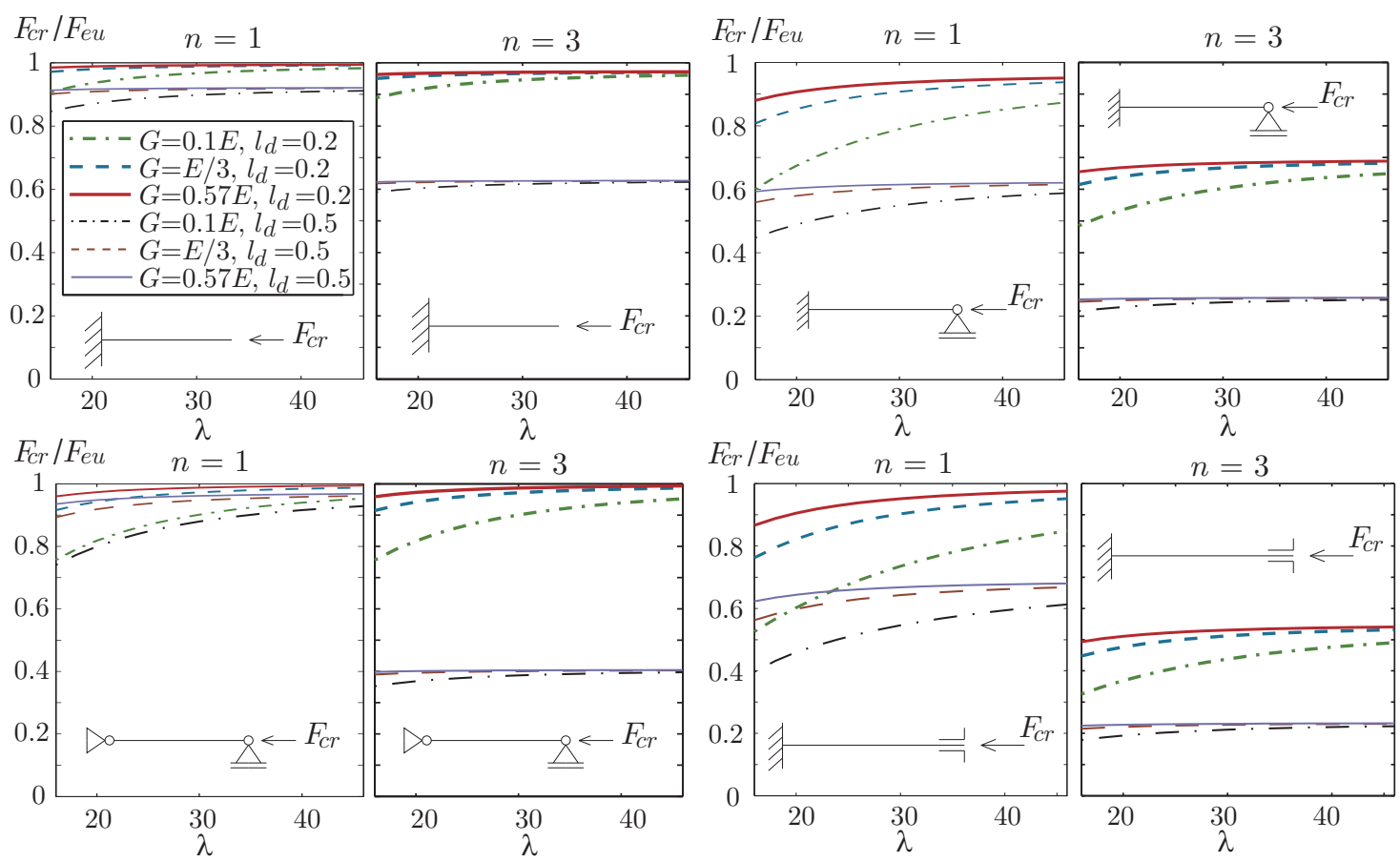

Fig. 8. Normalized buckling load vs. slenderness $\lambda$ for beams with one and three delaminations, for various shear-to-elastic modulus ratios and two delamination lengths, $l_{d}=0.2$ and 0.5 .

\subsection{Effect of the number of delaminations}

The comparison between Tables 2 and 3 indicates that the number of delaminations significally affects the normalized buckling load. In order to quantify this influence more systematically, the models with various delamination lengths $\left(l_{d}=0.2,0.4,0.6,0.8\right)$ were investigated in terms of the different number of delaminations, $n=1, \ldots, 5$. The following geometric and material properties of the beam were employed: $E=3 \cdot 10^{7} \mathrm{~N} / \mathrm{m}^{2}, G=E / 6, \lambda=32$. The laminae have equal thicknesses and are placed symmetrically in the longitudinal direction, therefore the vertical relative position of the $i$-th delamination is expressed as $r_{i}=\frac{i}{n+1}$. Results showing the dependency of the normalized buckling load on the number of delaminations are presented for various support conditions in Figs. 9, 10 and 11.

Fig. 9 shows that the increase of the number of short delaminations in simply supported and cantilever beams only slowly decreases the normalized buckling force. This changes for longer delaminations $\left(l_{d} \geq 0.4\right)$, where the effect of the number of delaminations becomes much more significant. For example, the buckling load for the simply supported beam with one delamination of length $l_{d}=0.6$ is $60 \%$ higher compared to the beam with two delaminations. It is 
also clear that the normalized buckling load rapidly decreases by the increase of the number of delaminations, if the delaminations are sufficiently long.

To analyze the effect of the delamination length and the number of delaminations in a greater detail, the buckling force vs. the delamination length is depicted in Fig. 10, for each number of delaminations separately. It is now clear that the increase of the length of delaminations grossly decreases the buckling force for multiple delaminations. This effect is most pronounced for the clamped-clamped beam and the least for the cantilever beam. In fact, for the cantilever having short delaminations $\left(l_{d}=0.2\right)$, there is no significant influence of the number of delaminations. By increasing both the length and the number of delaminations, the buckling load drops rapidly for

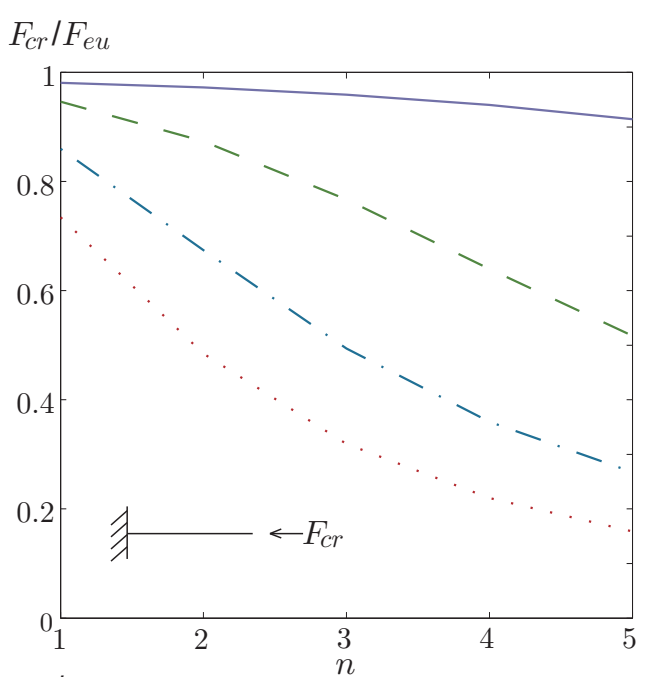

$F_{c r} / F_{e u}$
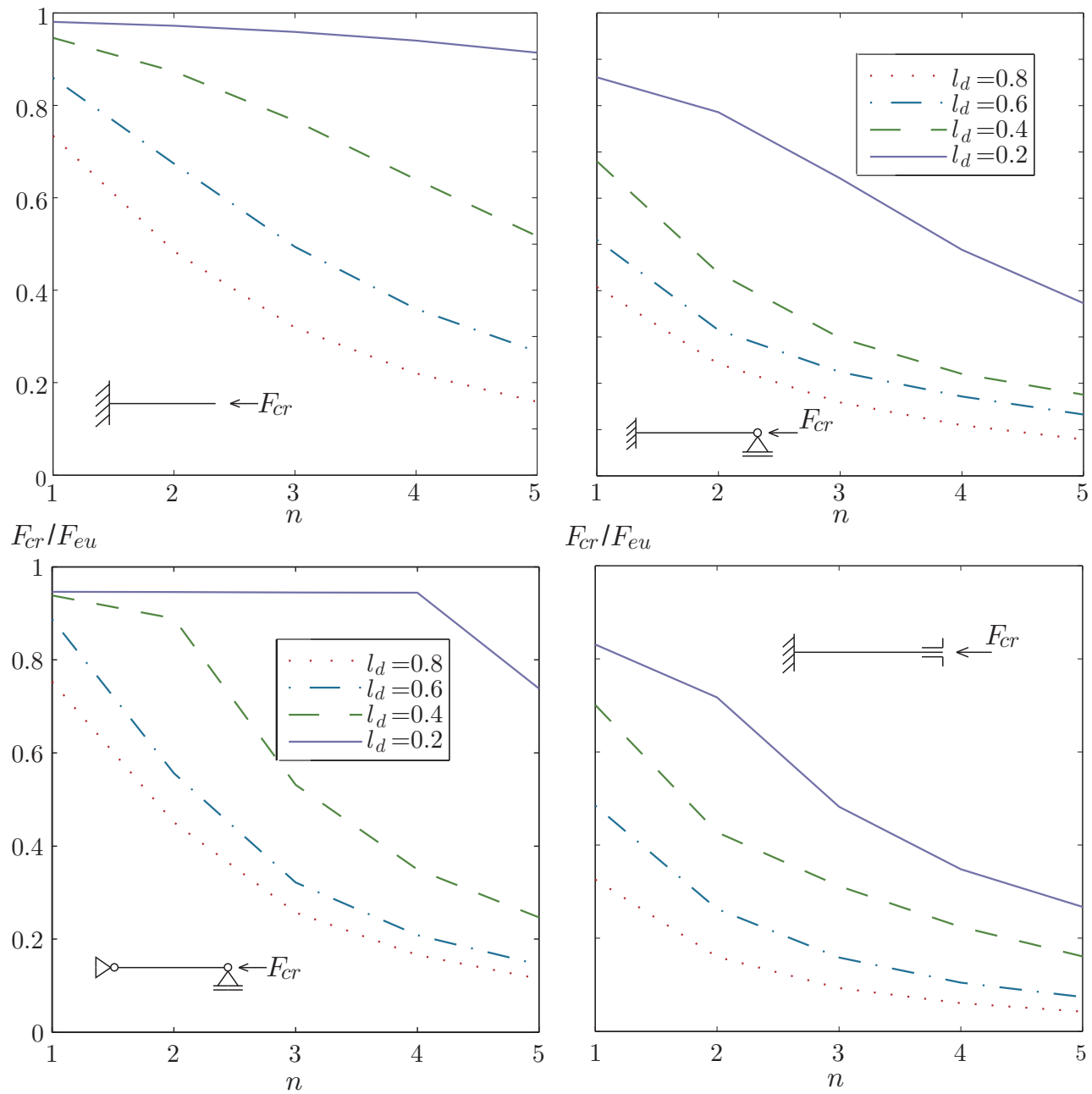

$F_{c r} / F_{e u}$

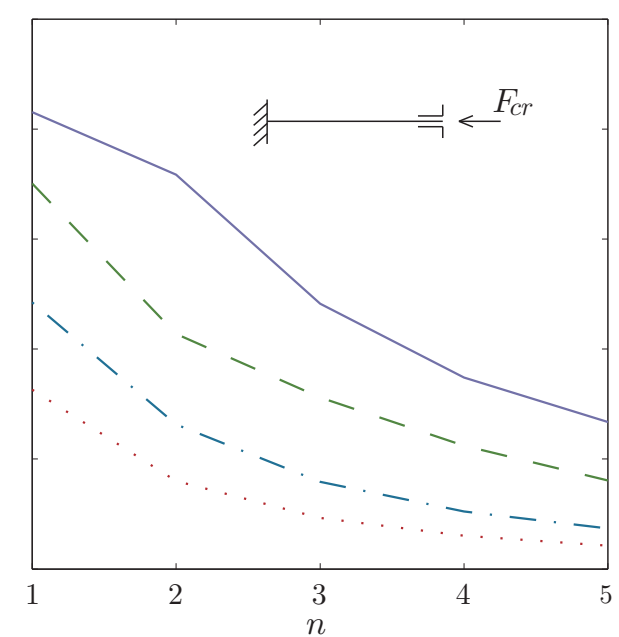

Fig. 9. Normalized buckling load vs. number of delaminations, $n$, for various lengths of delaminations, $l_{d}$. 


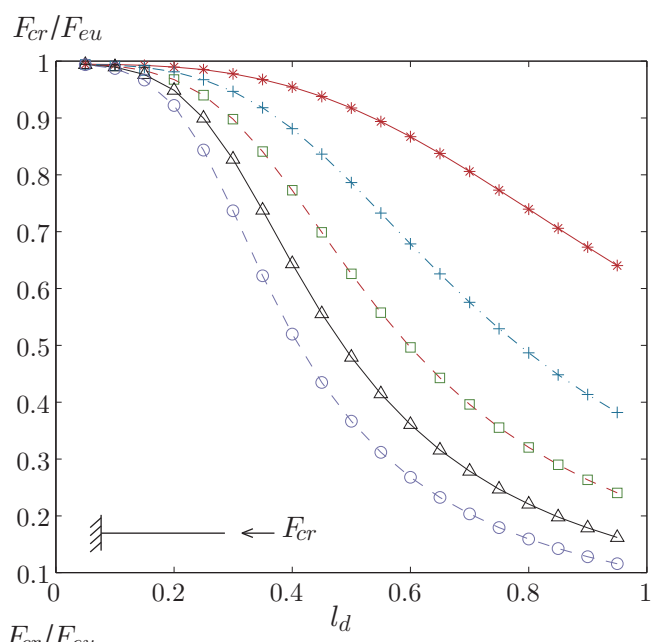

$F_{c r} / F_{e u}$
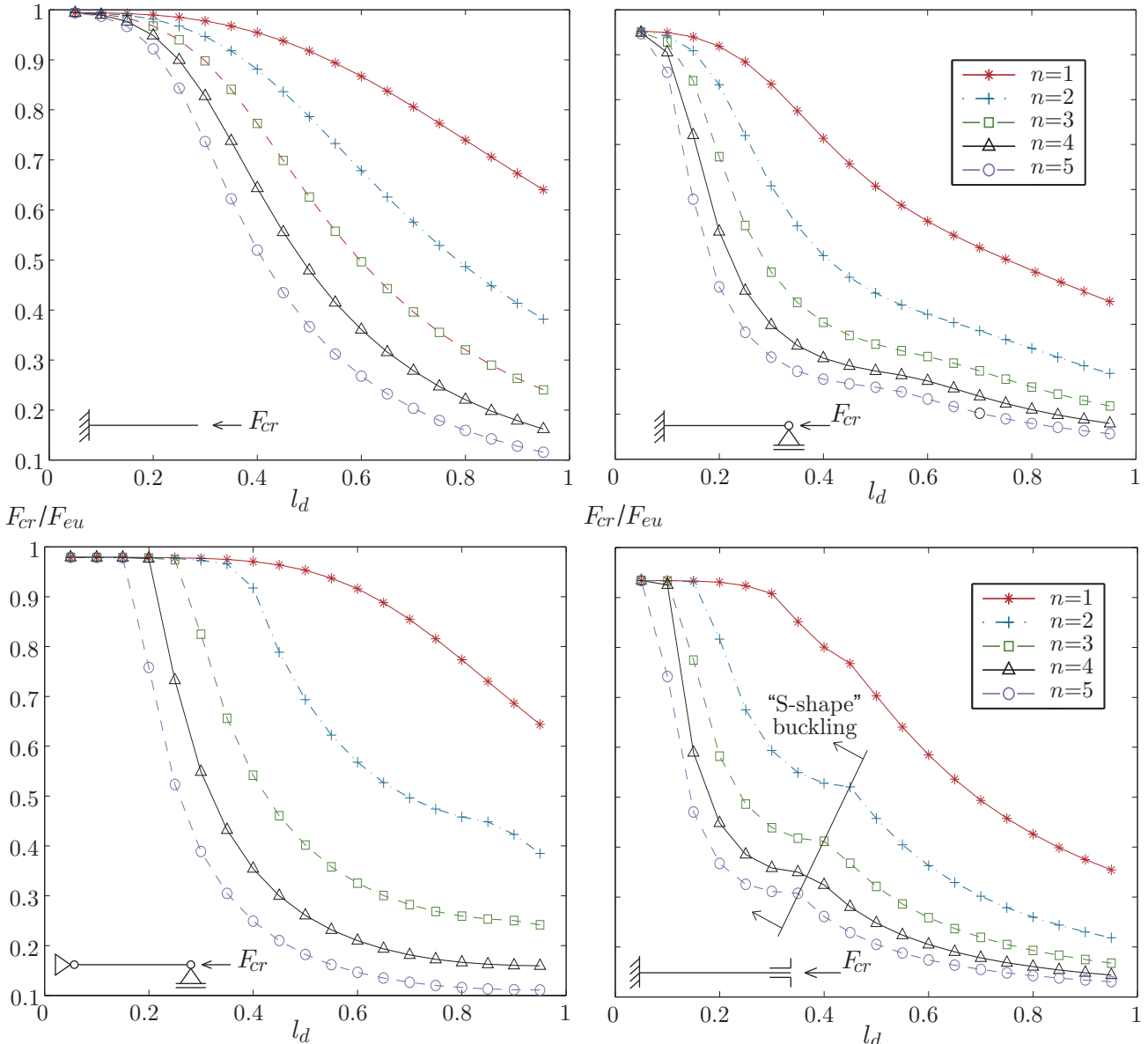

$F_{c r} / F_{e u}$

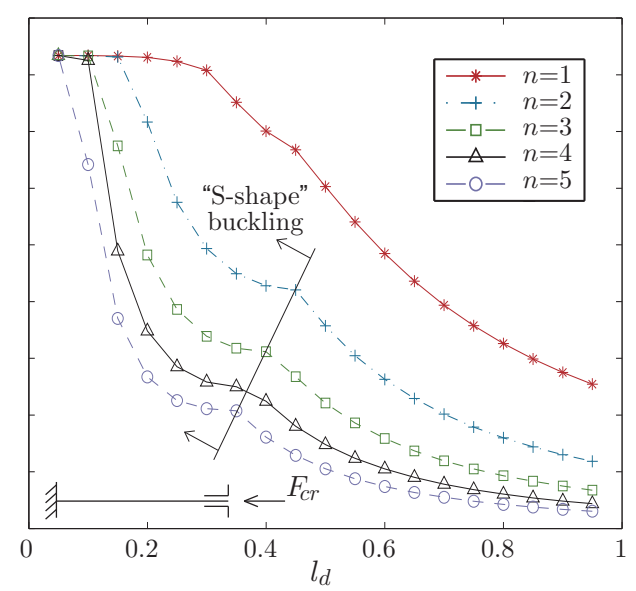

Fig. 10. Normalized buckling load vs. number of delaminations for several delamintion lengths.

the clamped-pinned and clamped-clamped beams. For example, the buckling forces of beams with two and three delaminations, differ for about $30 \%$. It is interesting to observe that the shapes of the $F_{c r}$ vs. $l_{d}$ curves for the clampedclamped beams differ from the shapes of the remaining curves, see Fig. 10 and the curves marked 'S-shape buckling' therein.

It is instructive to present the buckling load by a 3D graph representing the surface as a function of the number of the delaminations and the delamination length (Fig. 11). For the simply supported beam a flat region is formed in area of short delamination lengths $l_{d}$. There an increase of the delamination length has only a small influence on the buckling force. If the number of delaminations is increased, this area narrows, which eventually results in a rapid drop of the load. Such a flat plateau for short delaminations can also be observed with other support conditions, although the plateau is not so significant as in the simply supported beam. 

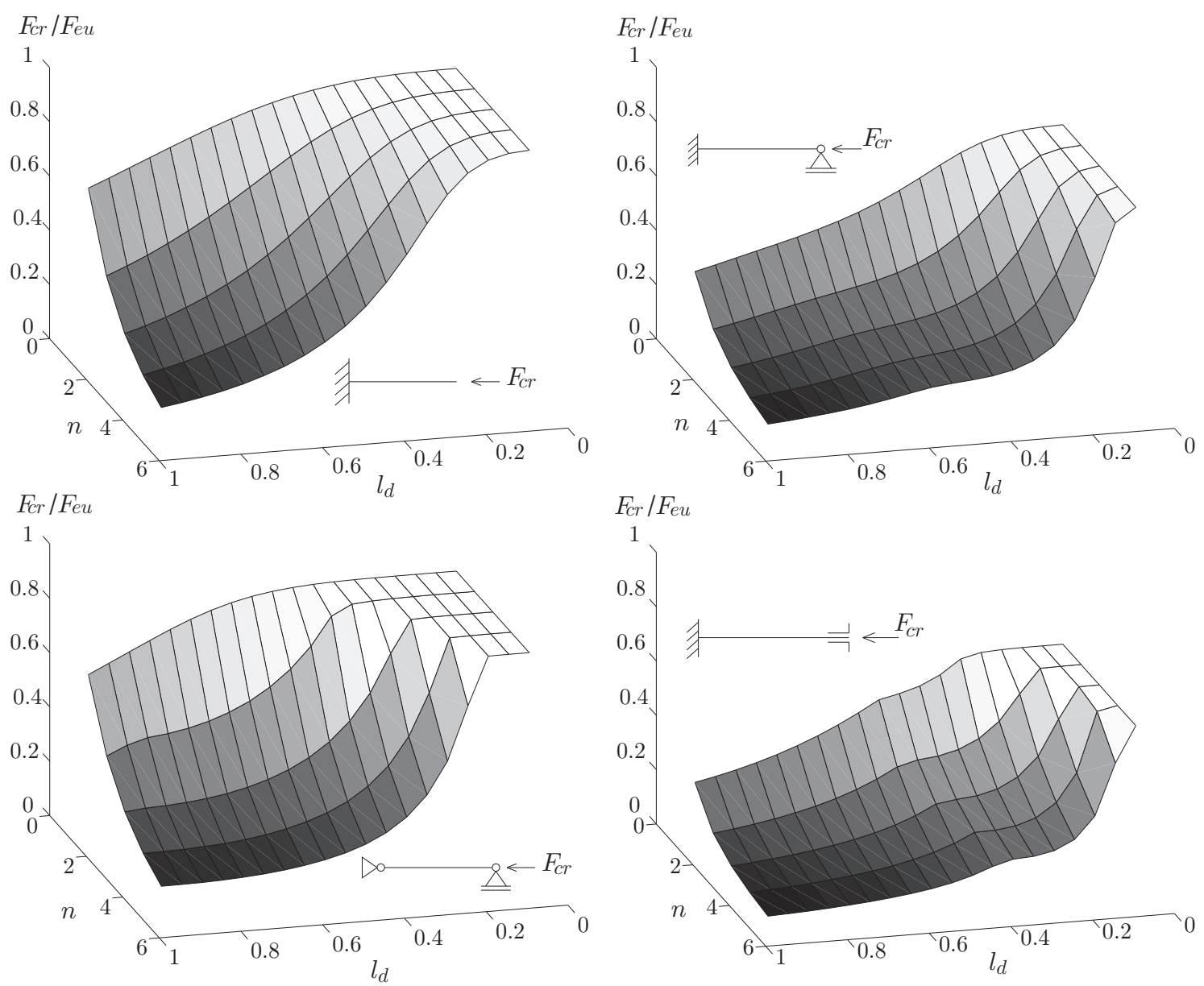

Fig. 11. Normalized buckling load vs. length of delamination $l_{d}$ and number of delaminations.

The graphs in Fig. 11 indicate that the cantilever is the most conservative with respect to the delamination length, in contrast to the clamped-clamped beam, which is strongly dependent on the delamination length. As already discussed in relation to Fig. 10, the so called 'S-shape' buckling takes place. Consequently, at some particular point, the increase of the delamination length does not decrease the buckling force significally. For further reading on the Sshaped-mode buckling see the paper by Parlapalli and Shu [18].

Thus the number of delaminations is insignificant if only short delaminations are expected to occur. In contrast, the effect of the number of layers is considerable for the beams with long delaminations. 
$(a)$

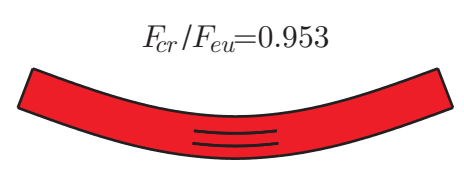

$l_{d}=0.2 \quad D_{0} \underline{\underline{R}} \leftarrow F_{c r}$

(b)

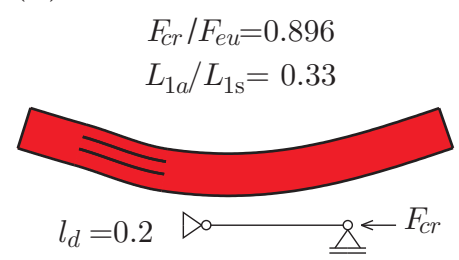

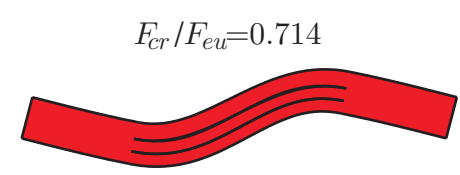
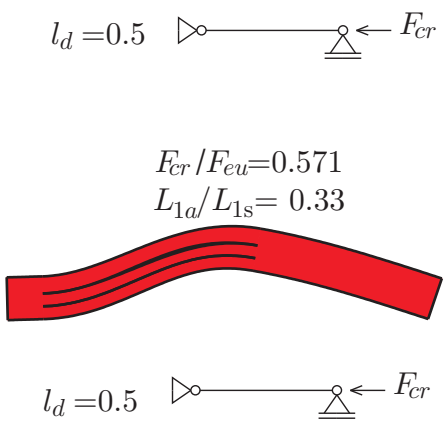

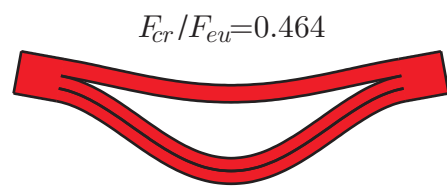

$l_{d}=0.8$ Do $\underline{\underline{\lambda}} \leftarrow F_{c r}$

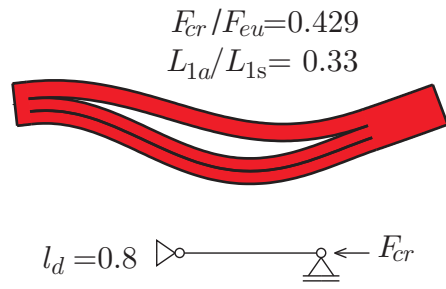

Fig. 12. Buckling shapes and normalized buckling forces for the simply-supported beam with two delaminations for various lengths, $l_{d}$. (a) symmetrical delamination; (b) asymmetrical delamination.

\subsection{Effect of the longitudinal eccentricity}

In order to demonstrate the effect of the longitudinal position of delaminations we have also investigated several models of beams with eccentrically positioned delaminations. The eccentricity is defined as the ratio between the length of the first element of the asymmetric and the related symmetric beam, $L_{1 a} / L_{1 s}$. We compared the results for simply supported, clamped-clamped and cantilever beams.

We first study the simply supported beam with two delaminations, which divide the beam into three laminae of equal thicknesses. Three different lengths of the delamination are examined. Material parameters employed in this study are typical for composite materials: $E=3 \cdot 10^{7} \mathrm{~N} / \mathrm{m}^{2}, G=E / 6$. The slenderness ratio of the beam is $\lambda=32$.

Fig. 12 displays the buckling shapes for both the longitudinally symmetrical and asymmetrical delaminations. The worsening effect of asymmetry is evident. The beams with longer delaminations buckle earlier. As observed from Fig. 12, not only the value of the buckling load but also the buckling shape is affected by the asymmetry. The highest discrepancies both in quantitative and qualitative respect appear at the middle-length delaminations, where the critical forces differ for about $20 \%$ and the 'S-shape' buckling mode typical for the symmetrical case almost disappears when asymmetry is taken into account. 
Next we present the results for stocky beams (the slenderness ratio being $\lambda=17$ ) and several support types. Fig. 13 shows the normalized buckling load vs. the delamination length for different longitudinal positions of delaminations for the cantilever, the simply supported and the clamped-clamped beam with one or three delaminations. For the cantilever the asymmetry of the boundary conditions results in asymmetric results with respect to the delamination position. The remaining support types give the results which are symmetric with respect to the midspan of the beam.
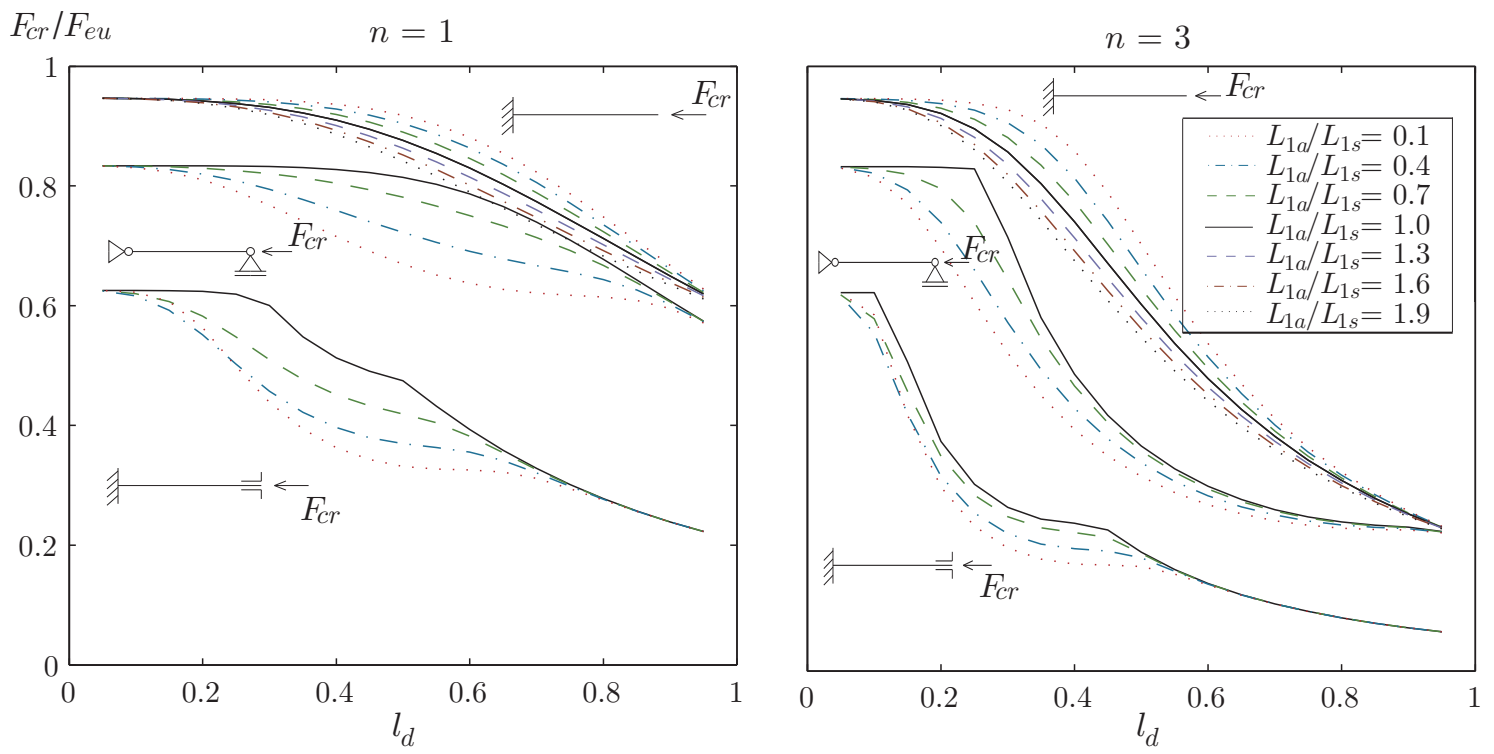

Fig. 13. Effect of longitudinal asymmetry on the normalized buckling load for the clamped-clamped beam, the simply supported beam and the cantilever with one or three delaminatons.

The longitudinal position of the delamination might cause a considerable reduction of the normalized buckling load. The largest effect is observed in short to middle-length delaminations $\left(0.2<l_{d}<0.6\right)$ for both single and multi-delaminated beams. Eccentricity of the delamination generally reduces the buckling load. The effect is the largest in simply supported beams, where the difference between the buckling force for symmetric and asymmetric delaminations grows to $15 \%$ for the beam with the delamination length $l_{d}=0.55$. Similar results are observed for the clamped-clamped beam with a single delamination, although the effect of its position is in this case bigger for shorter delaminations, $0.35<l_{d}<0.5$.

With the increase of the number of delaminations $(n=3)$ the effect of the longitudinal position of the delamination is higher for the cantilever. The buckling strength increases as the delamination moves toward the support and decreases when the delamination moves toward the free-end. For the simply supported beam with three delaminations, the largest influence of the delam- 
ination position is observed for $l_{d} \approx 0.25$, where the difference between the normalized forces for the symmetric and asymmetric cases is about $20 \%$. The multi-delaminated clamped-clamped beam shows a much smaller sensitivity to the delamination position.

\section{Conclusions}

We presented the exact analytical solution for the buckling load of the composite beam with multiple delaminations. The solution is based on Reissner's beam theory with a proper consideration of the extensional and bending stiffness coupling and the transverse shear effect. The presented model of the composite beam allows arbitrary longitudinal and vertical positions of delaminations. The exactness of the proposed method represents a solid base for studying the behaviour of various types of composite beams. Different positions, length of delaminations, slendernesses, shear moduli and the number of delaminations have substantial influence on the buckling behaviour. The effect of these parameters on the buckling loads was presented through systematic parametric studies. The essential results of these studies are:

(i) The dependence of the buckling load on the parameters is strongly nonlinear. Both behaviour and the value of the buckling load strongly depend on the way the beam is supported.

(ii) Increasing the delamination length not only reduces the relative buckling load but also affects the buckling shapes. The effect of the delamination length should be particularly considered with respect to the vertical position where the local buckling of laminae is dominant.

(iii) The shear effect can be substantial and may even not be neglected for isotropic-type of material, if the beams are stocky. For composite materials the shear effect is substantial for low to moderate slenderness ratios. The classical approach yield a very non-conservative results in such cases. Thus, it is recommended that for materials with high elastic-to-shear modulus ratio the shear effect is fully considered.

(iv) The increase of the number of middle-length to long delaminations results in a rapid reduction of the buckling loads. Typically, the 'S-shape' dependency on the delamination length for multi-delaminated clamped-clamped beam is observed. This is even more evident for a larger number of delaminations.

(v) The longitudinal asymmetry affects both the buckling load and the buckling shapes. The asymmetry could not be neglected, because the buckling load for the centric case could be overestimated by $20 \%$. 


\section{Acknowledgment}

This work was supported by the Slovenian Research Agency through the grant 1000-06-310187. The support is gratefully acknowledged.

\section{References}

[1] H. Chai, C. D. Babcock, W. B. Knauss, One dimensional modeling of failure in laminated plates by delamination buckling, International Journal of Solids and Structures 17, 1069-1083, 1981.

[2] H.-P. Chen, Shear deformation theory for compressive delamination buckling and growth, AIAA Journal 29, 813-819, 1991.

[3] H.-P. Chen, Transverse shear effects on buckling and postbuckling of laminated and delaminated plates, AIAA Journal 31, 163-169, 1993.

[4] B. Čas, M. Saje, I. Planinc, Non-linear finite element analysis of composite planar frames with an interlayer slip, Computers \& Structures 82, 1901-1912, 2004.

[5] B. Čas, M. Saje, I. Planinc, Buckling of layered wood columns, Advances in Engineering Software 38, 586-597, 2007.

[6] L. Euler, Methodus inveniendi lineas curvas maximi minimive proprietate gaudentes, Lausanne, 1744.

[7] H. Huang, G. A. Kardomateas, Buckling of orthotropic beam-plates with multiple delaminations, International Journal of Solids and Structures 35, 1355-1362, 1998.

[8] S. -F. Hwang, G. -H. Liu, Buckling behavior of composite laminates with multiple delaminations under uniaxial compression, Composite Structures 53, 235-243, 2001.

[9] G. A. Kardomateas, D. W. Schumueser, Buckling and post-buckling of delaminated composites under compressive loads including transverse shear effects, AIAA Journal 26, 337-343, 1988.

[10] H. B. Keller, Nonlinear bifurcation, Differential Equations 7, 417-434, 1970.

[11] N. Krauberger, M. Saje, I. Planinc, S. Bratina, Exact buckling load of a restrained $R C$ column, Structural Engineering and Mechanics 27, 1-17, 2007.

[12] Z. Kutlu, F. K. Chang, Modeling compressing failure of delaminated composites containing multiple through-the-width delaminations, Composite Materials 26, 350-387, 1992. 
[13] S. Lee, T. Park, G. Z. Voyiadjis, Free vibration analysis of axially compressed laminated composite beam-columns with multiple delaminations, Composites: Part B 33, 605-617, 2002.

[14] Q. S. Li, Effect of shear deformation on the critical buckling of multi-step bars, Structural Engineering and Mechanics 15, 71-81, 2003.

[15] Y. B. Lim, I. D. Parsons, The linearized buckling analysis of a composite beam with multiple delaminations, International Journal of Solids and Structures 30, 3085-3099, 1993.

[16] S. Moradi, F. Taheri, Delamination buckling analysis of general laminated composite beams by differential quadrature method, Composites: Part B 30, 503$511,1999$.

[17] K. S. Numayr, R. H. Haddad, Analytical Solution of Buckling of Beams with Two Delaminations, Mechanics of Composite Materials and Structures 8, 283297, 2001.

[18] M. R. Parlapalli, D. Shu, S-shaped mode in the lower and upper bounds of the buckling of composite beams with two equal delaminations, Composite Structures 81, 185-194, 2006.

[19] M. R. Parlapalli, D. Shu, Buckling of composite beams with two non-overlapping delaminations: Lower and Upper bounds, International Journal of Mechanical Sciences 49, 793-805, 2007.

[20] P. MSRao, D. Sylvain, D. Shu, C. N. Della, Buckling analysis of tri-layer beams with multiple separated delaminations, Composite Structures 66, 53-60, 2004.

[21] P. MSRao, D. Shu, Buckling analysis of two-layer beams with an asymmetric delamination, Engineering Structures 26, 651-658, 2004.

[22] P. MSRao, H. Song, D. Shu, Buckling analysis of tri-layered beams with overlapped delaminations, Journal of Composite Materials 39, 109-125, 2005.

[23] P. MS. Rao, T. Wenge, D. Shu, Buckling analysis of tri-layer beams with enveloped delaminations, Composites: part B 36, 33-39, 2005.

[24] E. Reissner, On one-dimensional finite-strain beam theory: The plane problem, Journal of Applied Mathematics Physics 23, 795-804, 1972.

[25] I. Sheinman, M. Soffer, Post-buckling analysis of composite delaminated beams, International Journal of Solids and Structures 27, 639-646, 1991.

[26] D. Shu, Buckling of multiple delaminated beams, International Journal of Solids and Structures 35, 1451-1465, 1998.

[27] H. Suemasu, Post-buckling behavior of composite panels with multiple delaminations, Composite Materials 27, 1077-1097, 1993.

[28] G. J. Simitses, S. Sallam, W. L. Yin, Effect of delamination of axially loaded homogeneous laminated plates, AIAA Journal 23, 1437-1444, 1985. 
[29] J.T.S. Wang, H.N. Pu, C.C. Lin, Buckling of Beam-Plates Having Multiple Delaminations, Journal of Composite Materials 31, 1002-1025, 1997.

[30] C. M. Wang, C. Y. Wang, J. N. Reddy, Exact Solutions for Buckling of Structural Members, CRC Press LLC, 2005.

[31] W. L. Yin, S. Sallam, G. J. Simitses, Ultimate axial load capacity of a delaminated beam-plate. AIAA Journal 24, 123-128, 1986.

[32] D. Zupan, M. Saje, The linearized three-dimensional beam theory of naturally curved and twisted beams: The strain vectors formulation, Computer Methods in Applied Mechanics and Engineering 195, 4557-4578, 2006. 